\title{
Effect of Patient Experience on Bypassing a Primary Care Gatekeeper: a Multicenter Prospective Cohort Study in Japan
}

\author{
Takuya Aoki, MD, MMA' ${ }^{7}$, Yosuke Yamamoto, $M D, P h D^{7}$, Tatsuyoshi lkenoue, $M D, M P H^{7}$, \\ Makoto Kaneko, MD 2,3, Morito Kise, MD2, Yasuki Fujinuma, $M D^{2}$, and Shunichi Fukuhara, MD, \\ DMSC, MACP $P^{1,4,5}$
}

\begin{abstract}
'Department of Healthcare Epidemiology, School of Public Health in the Graduate School of Medicine, Kyoto University, Kyoto, Japan; ${ }^{2}$ Centre for Family Medicine Development, Japanese Health and Welfare Co-operative Federation, Tokyo, Japan; ${ }^{3}$ Division of Clinical Epidemiology, Jikei University School of Medicine, Tokyo, Japan; ${ }^{4}$ Department of General Medicine, Shirakawa Satellite for Teaching And Research (STAR), Fukushima Medical University, Fukushima, Japan; ${ }^{5}$ Center for Innovative Research for Communities and Clinical Excellence (CIRC2LE), Fukushima Medical University, Fukushima, Japan.
\end{abstract}

BACKGROUND: To discuss how best to implement the gatekeeping functionality of primary care; identifying the factors that cause patients to bypass their primary care gatekeepers when seeking care should be beneficial.

OBJECTIVE: To examine the association between patient experience with their primary care physicians and bypassing them to directly obtain care from higher-level healthcare facilities.

DESIGN AND METHODS: This prospective cohort study was conducted in 13 primary care clinics in Japan. We assessed patient experience of primary care using the Japanese version of Primary Care Assessment Tool (JPCAT), which comprises six domains: first contact, longitudinality, coordination, comprehensiveness (services available), comprehensiveness (services provided), and community orientation. The primary outcome was the patient bypassing their usual primary care physician to seek care at a hospital, with this occurring at least once in a year. We used a Bayesian hierarchical model to adjust clustering within clinics and individual covariates.

KEY RESULTS: Data were analyzed from 205 patients for whom a physician at a clinic served as their usual primary care physician. The patient follow-up rate was $80.1 \%$. After adjustment for patients' sociodemographic and health status characteristics, the JPCAT total score was found to be inversely associated with patient bypass behavior (odds ratio per 1 SD increase, 0.44; 95\% credible interval, $0.21-0.88$ ). The results of various sensitivity analyses were consistent with those of the primary analysis.

CONCLUSIONS: We found that patient experience of primary care in Japan was inversely associated with bypassing a primary care gatekeeper to seek care at higher-level healthcare facilities, such as hospitals. Our findings suggest that primary care providers' efforts to improve patient experience should help to ensure

Electronic supplementary material The online version of this article (https://doi.org/10.1007/s11606-017-4245-1) contains supplementary material, which is available to authorized users.

Received May 3, 2017

Revised August 22, 2017

Accepted November 17, 2017

Published online January 19, 2018 appropriate use of healthcare services under loosely regulated gatekeeping systems; further studies are warranted.

KEY WORDS: gatekeeping; bypassing; primary care; patient experience; Japan.

J Gen Intern Med 33(5):722-8

DOI: $10.1007 / \mathrm{s} 11606-017-4245-1$

(c) Society of General Internal Medicine 2018

\section{INTRODUCTION}

Gatekeeping, defined as the process of matching patients' needs and preferences with the judicious use of medical services, is a role of the primary care system. Gatekeeping was developed to ensure the appropriate use of healthcare services and protect patients from the possible adverse effects of receiving unnecessary care. ${ }^{1,2}$ Conversely, strict gatekeeping is considered to be related to delayed diagnosis and adverse outcomes. ${ }^{3,4}$ One of the reasons why the level of gatekeeping varies widely among countries is the lack of evidence on the best methods to implement effective gatekeeping functionality. ${ }^{5}$ It ranges from patients having free access to specialists to mandates for obtaining a referral from a primary care physician (PCP) prior to accessing the services of a specialist.

In areas lacking primary care gatekeeping systems, many patients bypass lower-level healthcare facilities and directly seek care at a specialty care or hospital facility. ${ }^{6}$ Furthermore, under loosely regulated gatekeeping systems, patients may bypass PCPs as gatekeepers so as to visit specialty care or hospital facilities. ${ }^{7-9}$ Similar to the procedures based on economic incentives in France, Belgium, and Switzerland and point-of-service health insurance plans in the USA, Japan has adopted loosely regulated gatekeeping systems. ${ }^{5,9}$ In Japan, although referral from a PCP is not indispensable for consultation with specialists and hospitals, patients must pay additional costs to bypass their PCPs in clinics if they wish to receive care at higher-level healthcare facilities. Although Japanese patients are not required to register with a PCP, 
$53.7 \%$ of Japanese adults have PCPs acting as gatekeepers. ${ }^{10}$ Japanese PCPs provide preventive care and consultation under the national health insurance system to acutely and chronically ill patients on an outpatient basis or in their homes. In Japan, healthcare expenditures are paid on a fee-for-service basis.

To discuss how best to implement the gatekeeping functionality of primary care, identifying the factors that cause patients to bypass their primary care gatekeepers when seeking care should be beneficial. A few studies were conducted to investigate the factors associated with patient bypass of primary care gatekeepers. These studies suggested that a potential association exists between patient dissatisfaction with their PCP and their bypassing the PCP. ${ }^{7,8,11}$ However, these were exploratory studies that were limited by inadequate adjustment of confounders, and it is unclear whether an association exists between the patient experience, which is a more reliable measure of the patients' perception of healthcare quality than the patients' satisfaction, ${ }^{12}$ and the patients' bypass behavior. Patient satisfaction is subjective and often non-specific. In contrast, patient experience reflects actual experience, aiming to avoid value judgments and the effects of existing expectations. Patient experience is recognized as one of the three pillars of healthcare quality, along with clinical effectiveness and patient safety. ${ }^{13}$ This method is increasingly used to assess the quality of primary and higher-level care. ${ }^{14}$

In this study, we specifically aimed to examine the association between patient experience of primary care and bypassing a primary care gatekeeper to seek care at a higherlevel healthcare facility.

\section{METHODS}

\section{Setting and Participants}

We conducted a multicenter prospective cohort study in a primary care practice-based research network (PBRN) in Japan from October 2015 to October 2016. The ethics committee of the Kyoto University Graduate School of Medicine approved this study (approval no. R0184). PBRN is a group of ambulatory practices affiliated with each other that are principally devoted to the primary care of patients to investigate questions related to community-based practice. ${ }^{15}$ This PBRN is a nationally distributed network of primary care clinics belonging to the Japanese Health and Welfare Cooperative Federation. The 13 participating clinics are distributed into various medical areas, from urban to rural (the Tohoku, Kanto, Tokai, and Setouchi areas) (Appendix 1), with the majority of the clinics being operated by sole practitioners. Primary care provided at the clinics is delivered by family physicians, and the majority of these patients are elderly.

All outpatients aged 20 years and older who visited one of the participating clinics during the 3-day survey period in October 2015 were invited to participate in this study. Of the 1018 patients who were invited, $338(33.2 \%)$ agreed and completed baseline assessments using a self-administered

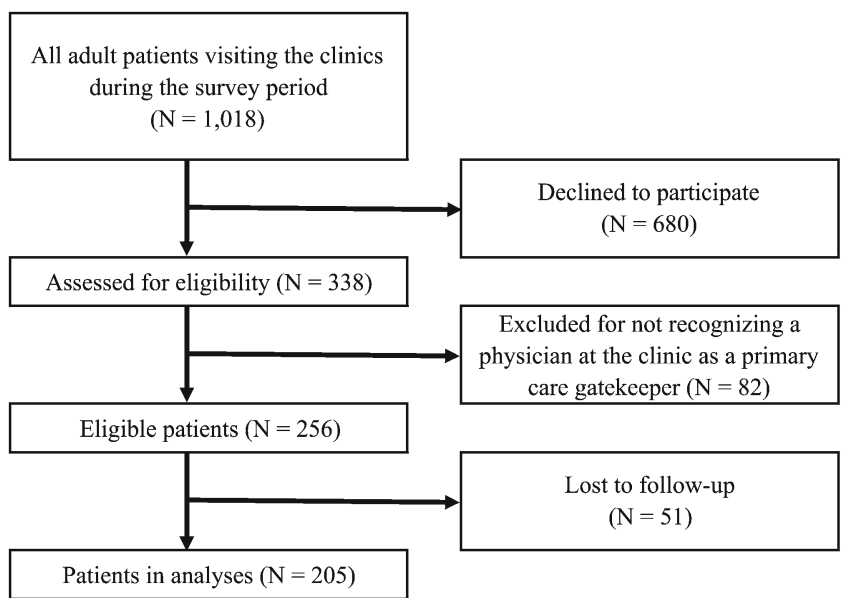

Figure 1 Patient flow chart

questionnaire (Fig. 1). The questionnaire measured the presence of the patient's usual PCP, the patient experience with his or her primary care, socioeconomic status, and health status characteristics. Among those who completed the initial survey, 256 patients were considered eligible based on having a physician at the clinic who serves as their usual primary care provider and functions as a gatekeeper. For this study, we used the same three questions in the Japanese version of Primary Care Assessment Tool (JPCAT) ${ }^{16}$ (Appendix 2) as the original Primary Care Assessment Tool adult expanded version (PCAT-AE) ${ }^{17}$ to identify a patient's usual PCP: (1) Is there a doctor that you usually visit if you are sick or need advice about your health? (usual source). (2) Is there a doctor who knows you best as a person? (knows best). (3) Is there a doctor who is most responsible for your health care? (most responsible). In this study, a patient was considered to have a usual PCP at the clinic if he or she positively answered all three questions and all three doctors were the same clinic physician.

A follow-up questionnaire was distributed 12 months after the completion of the initial survey to assess patient bypass behavior. In the follow-up survey, patients were considered lost to followup if they had switched a usual primary care physician within a follow-up period. We collected both the completed initial and follow-up surveys by mail. The patients who responded to the follow-up survey were given a small gift worth $500 \mathrm{JPY}$.

\section{MEASURES}

\section{Patient Experience of Primary Care}

We used JPCAT ${ }^{16}$ for data collection, which was based on PCAT-AE, ${ }^{17}$ to measure patient experience of primary care in Japan. This 29-item tool comprises six domains representing core primary care attributes: first contact, longitudinality, coordination, comprehensiveness (services available), comprehensiveness (services provided), and community orientation. ${ }^{18}$ The scoring system of JPCAT uses a 5-point Likert scale structured as follows: $1=$ strongly disagree, $2=$ somewhat disagree, $3=$ not sure, $4=$ somewhat agree, and $5=$ 
strongly agree. The responses are reduced by a factor of 1 and multiplied by 25 . The score for each of the domains is computed as the mean value for all converted scale scores within that domain. Thus, the domain scores range from 0 to 100 points, with higher scores indicating better performance. The total score is the mean of the six domain scores and reflects an overall measure of patient experience of primary care attributes. A previous study has shown that JPCAT has good reliability and validity for assessing these measures. ${ }^{16}$

\section{Bypass Behavior}

The primary outcome in this study was the patient bypassing their usual PCP to seek care at a higher-level healthcare facility. In this study, we specifically focused on the bypass behavior of patients who had a usual PCP. Thus, the patients' bypass behavior was defined as going directly to a hospital to seek care at least once in a year without having obtained a referral from their usual PCPs. Patients' bypass behavior as the primary outcome measure was determined by their responses to the follow-up survey question, "Did you ever visit a hospital directly without referral from your usual PCP when you have a health problem in the past year?" Participants were asked to answer on a binary scale ("yes" or "no"). If participants answered "yes" to the first question, they were then asked the following detailed questions on bypass behavior, "Did you ever visit a hospital directly without referral from your usual PCP on weekdays during the normal office hours of the clinics in the past year?" "Did you ever visit a hospital emergency department directly without referral from your usual PCP in the past year?" "Did you ever need hospitalization without referral from your usual PCP in the past year?" Participants were also asked on binary scale ("yes" or "no").

\section{Covariates}

Covariates were selected based on a clinical priority and a literature review to identify factors that may confound the association between patient experience of primary care and bypass behavior. ${ }^{8,19-25}$ We included covariates for sex, age, years of education, household income, duration of relationship with usual PCP, physical and mental health status, number of sources of care other than a usual PCP, and recent hospital admission. All covariates were evaluated using a selfadministered questionnaire. Physical and mental health statuses were measured using the 12-Item Short Form Health Survey Physical Component Summary and Mental Component Summary scores. ${ }^{26}$ Scores ranged from 0 to 100 , with higher scores indicating better health.

\section{STATISTICAL ANALYSIS}

Descriptive statistics for continuous data are reported as means and standard deviations, whereas categorical data are reported as frequencies and percentages. Student's t-test was used to analyze the continuous data, and the chi-square test was used to analyze the categorical data.

To determine whether the JPCAT total score was associated with patient bypass behavior, we used the Bayesian hierarchical model with a logit link function that includes a random effect for clinic and individual covariates as fixed effects. This model incorporated the random intercept and slope for a clinic using a centering within the cluster, because the threshold of bypass behavior and the influence of patient experience on bypass behavior were considered to differ by clinic size and regional variation. The JPCAT scores were centered around the clinic mean to estimate patient- and clinic-level effects separately; thus, we estimated the effect of patient experience on outcome within the same clinics. We fitted the Bayesian model with the Markov chain Monte Carlo algorithm using non-informative prior distribution and ran 2000 iterations for each of the four chains. The following individual covariates were included in the analysis: age, sex, years of education, household income, duration of relationship with usual PCP, physical and mental health status, number of other sources of care, and recent hospital admission. The mean and Bayesian 95\% credible interval (CI) were estimated and expressed as odds ratios (OR) for presentation. To allow for uncertainty in the missing values for independent variables, we used multiple imputations by fully conditional specification, with the JPCAT scores, sex, age, years of education, household income, duration of relationship with usual PCP, physical and mental health status, number of sources of care other than a usual PCP, and recent hospital admission as variables in the imputation model, thus creating five imputed data sets. We also carried out a case analysis of the patients with complete data on independent variables. In addition, we also performed exploratory analyses of the outcome in relation to each JPCAT domain score with the same model used for primary analyses.

To confirm the robustness of the study findings, sensitivity analyses were conducted using a different definition of bypass behavior. Because the primary outcome includes potential bypass behavior that may have occurred outside the normal office hours of the clinics, each model was reestimated with bypass behavior restricted to weekdays during the normal office hours of the clinics. In addition, we also conducted sensitivity analyses excluding patients who had required a hospital emergency department visit without a referral from their usual PCPs or hospitalization following bypass behavior.

We used R version 3.3.2 (R Foundation for Statistical Computing; https://www.r-project.org) and RStan version 2.14.1 (Stan Development Team; http://mc-stan.org) for statistical analyses.

\section{RESULTS}

Among the 256 eligible patients, 205 (80.1\%) completed the follow-up survey. There were no differences in sex, age, 
Table 1 Characteristics of Patients Who Completed Study vs. Those Lost to Follow-Up

\begin{tabular}{|c|c|c|c|}
\hline \multirow[t]{2}{*}{ Characteristic } & \multirow{2}{*}{$\begin{array}{l}\text { Completed } \\
(\mathrm{N}=\mathbf{2 0 5})\end{array}$} & \multirow{2}{*}{$\begin{array}{l}\begin{array}{l}\text { Lost to } \\
\text { follow-up }\end{array} \\
(N=51)\end{array}$} & \multirow[t]{2}{*}{$P$ value* } \\
\hline & & & \\
\hline \multicolumn{4}{|l|}{ Gender, N (\%) } \\
\hline Male & $100(49.8)$ & $23(45.1)$ & \multirow[t]{3}{*}{0.553} \\
\hline Female & $101(50.2)$ & $28(54.9)$ & \\
\hline Data missing & 4 & 0 & \\
\hline \multicolumn{4}{|l|}{ Age (years), N (\%) } \\
\hline $20-40$ & $3(1.5)$ & $0(0.0)$ & \multirow[t]{5}{*}{0.462} \\
\hline $41-60$ & $14(7.0)$ & $4(7.8)$ & \\
\hline $61-80$ & $149(74.1)$ & $36(70.6)$ & \\
\hline 81 or more & $35(17.4)$ & $11(21.6)$ & \\
\hline Data missing & 4 & 0 & \\
\hline \multicolumn{4}{|l|}{ Education, N (\%) } \\
\hline Less than high school & $45(23.3)$ & $15(30.0)$ & \multirow[t]{5}{*}{0.023} \\
\hline High school & $74(38.3)$ & $24(48.0)$ & \\
\hline Junior college & $30(15.5)$ & $7(14.0)$ & \\
\hline More than or equal to college & $44(22.8)$ & $4(8.0)$ & \\
\hline Data missing & 12 & 1 & \\
\hline \multicolumn{4}{|c|}{ Annual household income (million JPY), N (\%) } \\
\hline$<2.00(\fallingdotseq 18,000$ US dollar) & $48(25.9)$ & $16(34.8)$ & \multirow{4}{*}{0.105} \\
\hline $2.00-4.99$ & $100(54.1)$ & $25(54.3)$ & \\
\hline$\geqq 5.00$ & $37(20.0)$ & $5(10.9)$ & \\
\hline$\overline{\bar{D}}$ ata missing & 20 & 5 & \\
\hline \multicolumn{4}{|c|}{ Number of comorbidities $^{\dagger}, \mathrm{N}(\%)$} \\
\hline 0 & $11(5.5)$ & $0(0.0)$ & \multirow[t]{5}{*}{0.012} \\
\hline 1 & $46(23.1)$ & $8(15.7)$ & \\
\hline 2 & $56(28.1)$ & $12(23.5)$ & \\
\hline$\geqq 3$ & $86(43.2)$ & $31(60.8)$ & \\
\hline$\overline{\bar{D}}$ ata missing & 6 & 0 & \\
\hline SF-12 PCS, mean (SD) & $38.4(8.3)$ & $34.7(10.0)$ & \multirow[t]{2}{*}{0.008} \\
\hline Data missing & 18 & & \\
\hline SF-12 MCS, mean (SD) & $46.7(8.9)$ & $44.1(10.3)$ & \multirow{2}{*}{0.073} \\
\hline Data missing & 18 & & \\
\hline \multicolumn{4}{|c|}{ Duration of relationship with usual PCP (years), N (\%) } \\
\hline$<1$ & $6(3.0)$ & $0(0.0)$ & \multirow[t]{4}{*}{0.700} \\
\hline $1-5$ & $29(14.4)$ & $9(17.6)$ & \\
\hline $5<$ & $166(82.6)$ & $42(82.4)$ & \\
\hline Data missing & 4 & 0 & \\
\hline Number of other sources of care & $\mathrm{N}(\%)$ & & \\
\hline 0 & $81(40.5)$ & $15(30.0)$ & 0.046 \\
\hline 1 & $74(37.0)$ & $18(36.0)$ & \\
\hline 2 & $32(16.0)$ & $10(20.0)$ & \\
\hline$\geq 3$ & $13(6.5)$ & $7(14.0)$ & \\
\hline$\overline{\bar{D}}$ ata missing & 5 & 1 & \\
\hline Hospitalization in past 6 months, & $\mathrm{N}(\%)$ & & \\
\hline No & $173(92.5)$ & $45(93.8)$ & 0.768 \\
\hline Yes & $14(7.5)$ & $3(6.3)$ & \\
\hline Data missing & 18 & 3 & \\
\hline JPCAT scores, mean (SD) & & & \\
\hline Total score & $65.0(14.0)$ & $66.3(13.2)$ & 0.555 \\
\hline First contact & $46.3(24.5)$ & $43.8(23.4)$ & 0.516 \\
\hline Longitudinality & $79.4(15.1)$ & $84.7(13.3)$ & 0.023 \\
\hline Coordination & $70.0(26.1)$ & $70.8(23.6)$ & 0.829 \\
\hline Comprehensiveness & $70.0(21.0)$ & $73.3(19.6)$ & 0.340 \\
\hline (services available) & & & \\
\hline Comprehensiveness & $48.2(28.9)$ & $48.0(27.7)$ & 0.963 \\
\hline (services provided) & & & \\
\hline Community orientation & $75.0(15.6)$ & $76.6(15.6)$ & 0.495 \\
\hline
\end{tabular}

PCS, Physical Health Composite Scale score; MCS, Mental Health Composite Scale score

PCP, primary care physician; JPCAT, Japanese version of Primary Care Assessment Tool

$* P$ value by $t$-test for continuous data and chi-square test for categorical data

†Simple counts of the following chronic conditions: hypertension, diabetes, dyslipidemia, stroke, cardiac diseases, chronic respiratory diseases, digestive diseases, kidney diseases, urologic diseases, arthritis, rheumatism, mental disorders, endocrine diseases, and malignancy

thources of care other than a usual primary care physician
Table 2 Characteristics of Patients With or Without Bypassing a Primary Care Gatekeeper

\begin{tabular}{|c|c|c|c|}
\hline \multirow[t]{2}{*}{ Characteristic } & \multirow{2}{*}{$\begin{array}{l}\begin{array}{l}\text { Non- } \\
\text { bypassers }\end{array} \\
(N=177)\end{array}$} & \multirow{2}{*}{$\begin{array}{l}\text { Bypassers } \\
(N=28)\end{array}$} & \multirow[t]{2}{*}{$P$ value* } \\
\hline & & & \\
\hline \multicolumn{4}{|l|}{ Gender, N (\%) } \\
\hline Male & $83(48.0)$ & $17(60.7)$ & \multirow[t]{3}{*}{0.211} \\
\hline Female & $90(52.0)$ & $11(39.3)$ & \\
\hline Data missing & 4 & 0 & \\
\hline \multicolumn{4}{|l|}{ Age (years), N (\%) } \\
\hline $20-40$ & $2(1.2)$ & $1(3.6)$ & \multirow[t]{5}{*}{0.735} \\
\hline $41-60$ & $13(7.5)$ & $1(3.6)$ & \\
\hline $61-80$ & 129 (74.6) & $20(71.4)$ & \\
\hline 81 or more & $29(16.8)$ & $6(21.4)$ & \\
\hline Data missing & 4 & 0 & \\
\hline \multicolumn{4}{|l|}{ Education, N (\%) } \\
\hline Less than high school & $37(22.4)$ & $8(28.6)$ & \multirow[t]{5}{*}{0.496} \\
\hline High school & $63(38.2)$ & $11(39.3)$ & \\
\hline Junior college & $27(16.4)$ & $3(10.7)$ & \\
\hline More than or equal to college & $38(23.0)$ & $6(21.4)$ & \\
\hline Data missing & 12 & 0 & \\
\hline \multicolumn{4}{|c|}{ Annual household income (million JPY), N (\%) } \\
\hline$<2.00(\fallingdotseq 18,000$ US dollar $)$ & $41(25.9)$ & $7(25.9)$ & \multirow[t]{4}{*}{0.668} \\
\hline $2.00-4.99$ & $84(53.2)$ & $16(59.3)$ & \\
\hline$\geqq 5.00$ & $33(20.9)$ & $4(14.8)$ & \\
\hline $\bar{D}$ ata missing & 19 & 1 & \\
\hline \multicolumn{4}{|c|}{ Number of comorbidities ${ }^{\dagger}, \mathrm{N}(\%)$} \\
\hline 0 & $9(5.3)$ & $2(7.1)$ & \multirow[t]{5}{*}{0.160} \\
\hline 1 & $42(24.6)$ & $4(14.3)$ & \\
\hline 2 & $51(29.8)$ & $5(17.9)$ & \\
\hline$\geqq 3$ & $69(40.4)$ & $17(60.7)$ & \\
\hline $\bar{D}$ ata missing & 6 & 0 & \\
\hline SF-12 PCS, mean (SD) & $37.4(9.4)$ & $36.0(7.2)$ & \multirow[t]{2}{*}{0.508} \\
\hline Data missing & 17 & & \\
\hline SF-12 MCS, mean (SD) & $50.2(7.3)$ & $46.1(10.6)$ & \multirow{2}{*}{0.156} \\
\hline Data missing & 17 & & \\
\hline \multicolumn{4}{|c|}{ Duration of relationship with usual PCP (years), N (\%) } \\
\hline$<1$ & $6(3.5)$ & $0(0.0)$ & \multirow[t]{4}{*}{0.242} \\
\hline $1-5$ & $26(15.0)$ & $3(10.7)$ & \\
\hline $5<$ & $141(81.5)$ & $25(89.3)$ & \\
\hline Data missing & 4 & 0 & \\
\hline Number of other sources of care & $\mathrm{N}(\%)$ & & \\
\hline 0 & $72(41.9)$ & $9(32.1)$ & 0.038 \\
\hline 1 & $66(38.4)$ & $8(28.6)$ & \\
\hline 2 & $25(14.5)$ & $7(25.0)$ & \\
\hline$\geqq 3$ & $9(5.2)$ & $4(14.3)$ & \\
\hline $\bar{D}$ ata missing & 5 & 0 & \\
\hline Hospitalization in past 6 months, & $\mathrm{N}(\%)$ & & \\
\hline No & $148(91.4)$ & $25(100.0)$ & 0.126 \\
\hline Yes & $14(8.6)$ & $0(0.0)$ & \\
\hline Data missing & 15 & 3 & \\
\hline JPCAT scores, mean (SD) & & & \\
\hline Total score & $65.7(13.7)$ & $60.5(15.2)$ & 0.066 \\
\hline First contact & $47.4(24.6)$ & $39.6(23.6)$ & 0.120 \\
\hline Longitudinality & $79.6(14.9)$ & $78.0(16.5)$ & 0.591 \\
\hline Coordination & $70.4(25.9)$ & $67.0(27.8)$ & 0.515 \\
\hline Comprehensiveness & $71.2(20.8)$ & $63.2(21.7)$ & 0.063 \\
\hline (services available) & & & \\
\hline Comprehensiveness & $49.3(28.6)$ & $41.9(30.2)$ & 0.224 \\
\hline (services provided) & & & \\
\hline Community orientation & $75.4(15.4)$ & $72.4(16.8)$ & 0.361 \\
\hline
\end{tabular}

PCS, Physical Health Composite Scale score; MCS, Mental Health Composite Scale score

PCP, primary care physician; JPCAT, Japanese version of Primary Care Assessment Tool

$* P$ value by $t$-test for continuous data and chi-square test for categorical data

†Simple counts of the following chronic conditions: hypertension, diabetes, dyslipidemia, stroke, cardiac diseases, chronic respiratory diseases, digestive diseases, kidney diseases, urologic diseases, arthritis, rheumatism, mental disorders, endocrine diseases, and malignancy $\neq$ Sources of care other than a usual primary care physician 
household income, mental health status, recent hospital admission, and JPCAT total scores between the 51 subjects who had been lost to follow-up and those who had completed follow-up (Table 1). However, we noted a trend suggesting that subjects who were lost to follow-up had fewer years of education, more comorbidities and sources of care, and worse physical health status compared with those who completed follow-up.

Table 2 compares the demographic information of the patients in the bypass group with that of the patients who had not bypassed their usual PCPs. We noted a trend suggesting that the bypass group had lower JPCAT total scores (mean difference, 5.2 points). Among the patients in the bypass group, 25 (89.3\%) had bypassed their usual PCPs on weekdays during the normal office hours of the clinics, 4 (14.3\%) had visited hospital emergency departments without referral from their usual PCPs, and 3 (10.7\%) had required hospitalization following bypass.

Figure 2 shows the results of multivariable analyses using the Bayesian hierarchical model and the multiple imputation to investigate the association of the JPCAT total scores with bypass behavior. After adjustment for possible confounders and clustering within clinics, we found that the JPCAT total score was inversely associated with bypass behavior (OR per 1 SD increase, $0.44 ; 95 \%$ CI, 0.21-0.88).

The results of complete case analyses showed no significant differences from the findings using multiple imputations. Sensitivity analyses using a definition of bypass behavior as having occurred only during normal office hours or excluding patients who had required a hospital emergency department visit or hospitalization showed results similar to those of the primary analysis.

\section{DISCUSSION}

The results of the multivariable analysis with adjustment for possible confounders and cluster effects showed that the JPCAT total score was inversely associated with bypassing a primary care gatekeeper in favor of receiving higher level care at a hospital. When the sensitivity analysis was conducted using a more stringent definition of bypass behavior, the bypass OR for the JPCAT total score was found to be similar to OR derived from the primary analysis. Furthermore, only a few patients from the bypass group had required hospitalization. Overall, our results showed that better patient experience of primary care acts to suppress bypass behavior in Japan. Our findings reinforced the significance of patient experience of primary care in terms of the appropriate use of healthcare services under loosely regulated gatekeeping systems.

Several studies have been conducted to investigate the factors associated with patients' bypass behavior in the geographic context of developing countries and rural areas. ${ }^{20,23,24}$ In these studies, bypass behavior was defined as patients receiving health care from providers located farther away from their homes than the nearest healthcare providers. Despite the fact that bypass behavior within the context of a patient's relationship with a PCP is also a crucial determinant of the excessive care and cost increase, bypass in this context has not been researched adequately. According to previous studies, patient experience affects various behaviors, such as adherence to treatment, cancer prevention activities, emergency department visits, and advance care planning. ${ }^{27-30}$ The results of this study have contributed additional findings on the association between patient experience and healthcare utilization.

This is the first confirmatory longitudinal study to reveal the association between patient experience of primary care and bypassing a primary care gatekeeper to seek care at a higher-

\begin{tabular}{lc}
\hline Scale & Adjusted OR (95\% CI) \\
\hline JPCAT total score & $0.44(0.21-0.88)$ \\
& \\
JPCAT domain scores & \\
First contact & $0.62(0.29-1.32)$ \\
Longitudinality & $0.85(0.46-1.57)$ \\
Coordination & $0.59(0.26-1.20)$ \\
Comprehensiveness (services available) & $0.62(0.34-1.08)$ \\
Comprehensiveness (services provided) & $0.52(0.19-1.17)$ \\
Community orientation & $0.78(0.41-1.48)$
\end{tabular}

Figure 2 Factors associated with bypassing a primary care gatekeeper $(N=205)$. JPCAT, Japanese version of Primary Care Assessment Tool; OR, odds ratio; CI, credible interval; Error bars indicate 95\% CIs. Bayesian hierarchical modeling (random slope model). Adjusted for age, sex, education, household income, duration of relationship with usual primary care physician, number of other sources of care, SF-12 PCS, SF12 MCS, and hospitalization in past 6 months. OR per 1 SD increase 
level healthcare facility. Patient experience is the most reliable measure of a patient's perception of healthcare quality, and PCAT is an internationally established measure for the evaluation of patient experience of primary care. Our findings were based on the data from a nationwide multicenter PBRN study covering both urban and rural areas and included a wide range of local healthcare delivery systems. The influence of patient experience on bypass behavior was considered to differ by clinic and geographic area; therefore, we adjusted for clustering within clinics using a Bayesian hierarchical model (random slope model) and allowed for appropriate patient-level analysis.

Our study had several limitations. First, the participation rate was a concern. In the case of patient experience surveys, a previous study showed that a low participation rate did not introduce selective non-response bias $^{31}$; however, there is a possibility that patients with low patient experience levels were less likely to respond to our survey. Furthermore, we had a moderate followup rate of $80.1 \%$. We did not have complete outcome data on patients who had lost their ability to respond to the survey or who had died during the follow-up period. There was no significant difference in the JPCAT total scores between the patients who were lost to follow-up and those who completed it. Although we did not have data on the outcomes of the patients who were lost to follow-up, they might bypass their usual PCPs more frequently than those who completed follow-up because of social desirability bias. ${ }^{32}$ Although the fact that the follow-up survey was sent to patients through the mail could also have resulted in some of the patients having been lost to follow-up, we did not follow up with the patients in each clinic directly to avoid interviewer bias. Second, we did not adjust for clustering within physicians in the analyses. However, the impact of this on our study's results may be limited because the majority of participating clinics were managed by one full-time physician. Third, our survey setting was restricted to primary care clinics that had a known interest in healthcare quality. Although we covered a nationally distributed network of primary care clinics, caution should be exercised when generalizing the results of this study. Because our study population was limited to patients having a usual PCP and the majority of the patients were elderly, the generalizability of our findings to other populations may be limited.

\section{CONCLUSIONS}

We found that patient experience of primary care in Japan was inversely associated with bypassing a primary care gatekeeper to receive higher-level care at a hospital. Our findings suggest that primary care providers' efforts to improve patient experience should help to ensure the appropriate use of healthcare services under loosely regulated gatekeeping systems; further studies are warranted.

Acknowledgements: The authors thank the participating primary care clinics belonging to the Japanese Health and Welfare Cooperative Federation. This work was supported by the Pfizer Health Research Foundation, Japan (grant no. 15-9-051).

Corresponding Author: Shunichi Fukuhara, MD, DMSc, MACP; Department of Healthcare Epidemiology, School of Public Health in the Graduate School of MedicineKyoto University, Kyoto, Japan (e-mail: fukuhara.shunichi.6m@kyoto-u.ac.jp).

\section{Compliance with Ethical Standards:}

Conflict of Interest: The authors declare that they do not have a conflict of interest.

\section{REFERENCES}

1. Franks $\mathbf{P}$, Clancy CM, Nutting PA. Gatekeeping revisited-protecting patients from overtreatment. N Engl J Med. 1992;327(6):424-9.

2. Velasco Garrido M, Zentner A, Busse R. The effects of gatekeeping: a systematic review of the literature. Scand J Prim Health Care 2011;29(1):28-38.

3. Young GP, Lowe RA. Adverse outcomes of managed care gatekeeping. Acad Emerg Med. 1997;4(12):1129-36.

4. Vedsted $\mathbf{P}$, Olesen $\mathbf{F}$. Are the serious problems in cancer survival partly rooted in gatekeeper principles? An ecologic study. Br J Gen Pract 2011;61(589):508-12.

5. Greenfield G, Foley $\mathbf{K}$, Majeed A. Rethinking primary care's gatekeeper role. BMJ. 2016;4803(September):i4803.

6. Wu D, Wang Y, Lam KF, Hesketh T. Health system reforms, violence against doctors and job satisfaction in the medical profession: a crosssectional survey in Zhejiang Province, Eastern China. BMJ Open. 2014;4(12):e006431.

7. Kulu-Glasgow I, Delnoij D, de Bakker D. Self-referral in a gatekeeping system: patients' reasons for skipping the general-practitioner. Health Policy. 1998;45(3):221-38.

8. Tabenkin H, Gross R, Brammli S, et al. Patients' views of direct access to specialists: an Israeli experience. JAMA. 1998;279(24):1943-8.

9. Forrest CB, Weiner JP, Fowles J, et al. Self-referral in point-of-service health plans. JAMA. 2001;285(17):2223-31.

10. Japan Medical Association Research Institute. The 5th survey on attitudes towards healthcare in Japan. Available at: http://www.jmari. med.or.jp/download/WP331.pdf. Accessed April 2, 2017.

11. Braun BL, Fowles JB, Forrest CB, Kind EA, Foldes SS, Weiner JP. Which enrollees bypass their gatekeepers in a plan? Med Care. 2003;41(7):836-41.

12. Salisbury C, Wallace M, Montgomery AA. Patients' experience and satisfaction in primary care: secondary analysis using multilevel modelling. BMJ Br Med J. 2010;341(7777):820.

13. Institute of Medicine. Committee on Quality of Health Care in America. Crossing the quality chasm: A new health system for the 21 st century. Washington DC: National Academies Press; 2001.

14. Browne K, Roseman D, Shaller D, Edgman-Levitan S. Analysis \& commentary. Measuring patient experience as a strategy for improving primary care. Health Affairs 2010;29:921-5.

15. AHRQ. Primary Care Practice-based Research Networks. Available at: http://www.ahrq.gov/sites/default/files/publications/files/pbrn.pdf. Accessed April 2, 2017.

16. Aoki T, Inoue M, Nakayama T. Development and validation of the Japanese version of Primary Care Assessment Tool. Fam Pract 2016;33 (1):112-7.

17. Shi L, Starfield B, Xu J. Validating the Adult Primary Care Assessment Tool. J Fam Pract 2001;50 (2): 161-75.

18. Starfield B. Primary care: balancing health needs, services, and technology. Oxford, UK: Oxford University Press; 1998.

19. Campbell JL, Ramsay J, Green J. Age, gender, socioeconomic, and ethnic differences in patients' assessments of primary health care. Qual Health Care. 2001;10(2):90-5. 
20. Liu J, Bellamy G, Barnet B, Weng S. Bypass of local primary care in rural counties: Effect of patient and community characteristics. Ann Fam Med. 2008;6(2):124-30.

21. Xiao H, Barber JP. The effect of perceived health status on patient satisfaction. Value Heal. 2008;11(4):719-25.

22. Lyratzopoulos G, Elliott M, Barbiere JM, et al. Understanding ethnic and other socio-demographic differences in patient experience of primary care: evidence from the English General Practice Patient Survey. BMJ Qual Saf. 2012;21(1):21-9.

23. Yaffee AQ, Whiteside LK, Oteng RA, et al. Bypassing proximal health care facilities for acute care: a survey of patients in a Ghanaian Accident and Emergency Centre. Trop Med Int Heal. 2012;17(6):775-81.

24. Sanders SR, Erickson LD, Call VRA, Mcknight ML, Hedges DW. Rural health care bypass behavior: How community and spatial characteristics affect primary health care selection. J Rural Heal. 2015;31(2):146-56.

25. Sizmur S, Graham C, Walsh J. Influence of patients' age and sex and the mode of administration on results from the NHS Friends and Family Test of patient experience. J Health Serv Res Policy. 2015;20(1):5-10.
26. Ware JJ, Kosinski MM, Keller SSD. A 12-Item Short-Form Health Survey: construction of scales and preliminary tests of reliability and validity. Med Care. 1996;34(3):220-33.

27. Safran DG, Taira DA, Rogers WH, Kosinski M, Ware JE, Tarlov AR. Linking primary care performance to outcomes of care. J Fam Pract. 1998;47(3):213-20.

28. Gary TL, Maiese EM, Batts-Turner M, Wang NY, Brancati FL. Patient satisfaction, preventive services, and emergency room use among African-Americans with type 2 diabetes. Dis Manag. 2005;8(6):361-71.

29. Aoki T, Inoue M. Primary care patient experience and cancer screening uptake among women: an exploratory cross-sectional study in a Japanese population. Asia Pac Fam Med. 2017;16(1):3.

30. Aoki T, Miyashita J, Yamamoto $\mathbf{Y}$, et al. Patient experience of primary care and advance care planning: a multicentre cross-sectional study in Japan. Fam Pract. 2017;34(2):206-12.

31. Roland M, Elliott M, Lyratzopoulos G et al. Reliability of patient responses in pay for performance schemes: analysis of national General Practitioner Patient Survey data in England. BMJ 2009;339:b3851.

32. Bader F, Bauer J, Kroher M, Riordan P. Privacy Concerns in responses to sensitive questions. A survey experiment on the influence of numeric codes on unit nonresponse, item nonresponse, and misreporting. Methods, Data, Anal. 2016;10(1):47-72. 